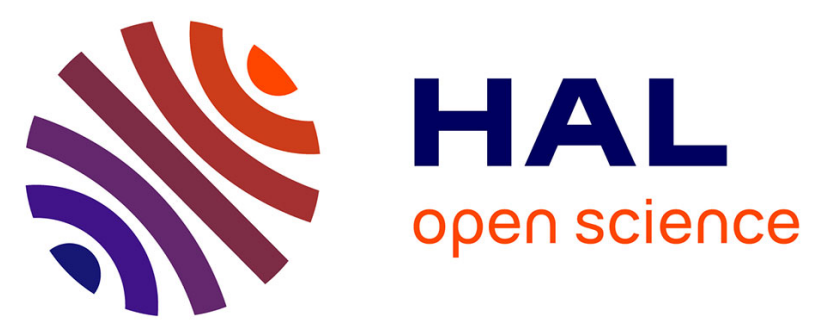

\title{
Sunward-propagating Whistler Waves Collocated with Localized Magnetic Field Holes in the Solar Wind: Parker Solar Probe Observations at 35.7 R Radii
}

O.V. Agapitov, Thierry Dudok de Wit, F. S. Mozer, J.W. Bonnell, J.F. Drake, D. Malaspina, V.V. Krasnoselskikh, S. Bale, P. Whittlesey, A. W. Case, et al.

\section{To cite this version:}

O.V. Agapitov, Thierry Dudok de Wit, F. S. Mozer, J.W. Bonnell, J.F. Drake, et al.. Sunwardpropagating Whistler Waves Collocated with Localized Magnetic Field Holes in the Solar Wind: Parker Solar Probe Observations at 35.7 R Radii. The Astrophysical journal letters, 2020, 891 (1), pp.L20. 10.3847/2041-8213/ab799c . insu-02517676

\section{HAL Id: insu-02517676 \\ https://hal-insu.archives-ouvertes.fr/insu-02517676}

Submitted on 6 Apr 2020

HAL is a multi-disciplinary open access archive for the deposit and dissemination of scientific research documents, whether they are published or not. The documents may come from teaching and research institutions in France or abroad, or from public or private research centers.
L'archive ouverte pluridisciplinaire HAL, est destinée au dépôt et à la diffusion de documents scientifiques de niveau recherche, publiés ou non, émanant des établissements d'enseignement et de recherche français ou étrangers, des laboratoires publics ou privés. 


\title{
Sunward-propagating Whistler Waves Collocated with Localized Magnetic Field Holes in the Solar Wind: Parker Solar Probe Observations at $35.7 \boldsymbol{R}_{\odot}$ Radii
}

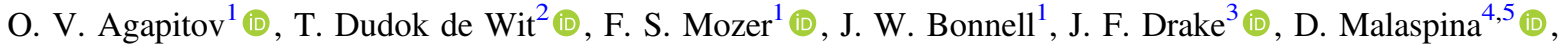

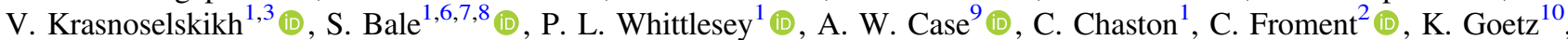

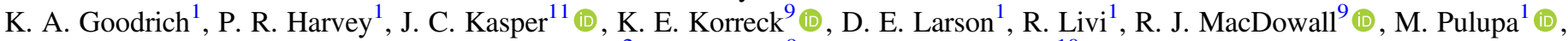 \\ C. Revillet $^{2}$, M. Stevens ${ }^{9}$ (D), and J. R. Wygant ${ }^{10}$ \\ ${ }^{1}$ Space Sciences Laboratory, University of California, Berkeley, CA 94720, USA; agapitov@ssl.berkeley.edu \\ ${ }^{2}$ LPC2E/CNRS-University of Orléans, Orléans, France \\ ${ }^{3}$ University of Maryland, College Park, MD, USA \\ ${ }^{4}$ Laboratory for Atmospheric and Space Physics, University of Colorado, Boulder, CO, USA \\ ${ }^{5}$ Astrophysical and Planetary Sciences Department, University of Colorado, Boulder, CO, USA \\ ${ }^{6}$ Physics Department, University of California, Berkeley, CA, USA \\ ${ }^{7}$ The Blackett Laboratory, Imperial College London, London, UK \\ ${ }^{8}$ School of Physics and Astronomy, Queen Mary University of London, London, UK \\ ${ }^{9}$ Harvard-Smithsonian Center for Astrophysics, Cambridge, MA, USA \\ ${ }^{10}$ University of Minnesota, Minneapolis, MN, USA \\ ${ }^{11}$ University of Michigan, Ann Arbor, MI, USA \\ Received 2020 January 30; revised 2020 February 12; accepted 2020 February 20; published 2020 March 4
}

\begin{abstract}
Observations by the Parker Solar Probe mission of the solar wind at $\sim 35.7$ solar radii reveal the existence of whistler wave packets with frequencies below $0.1 f_{\text {ce }}(20-80 \mathrm{~Hz}$ in the spacecraft frame). These waves often coincide with local minima of the magnetic field magnitude or with sudden deflections of the magnetic field that are called switchbacks. Their sunward propagation leads to a significant Doppler frequency downshift from $200-300$ to $20-80 \mathrm{~Hz}$ (from 0.2 to $0.5 f_{\text {ce }}$ ). The polarization of these waves varies from quasi-parallel to significantly oblique with wave normal angles that are close to the resonance cone. Their peak amplitude can be as large as $2-4 \mathrm{nT}$. Such values represent approximately $10 \%$ of the background magnetic field, which is considerably more than what is observed at $1 \mathrm{au}$. Recent numerical studies show that such waves may potentially play a key role in breaking the heat flux and scattering the Strahl population of suprathermal electrons into a halo population.
\end{abstract}

Unified Astronomy Thesaurus concepts: Solar wind (1534)

\section{Introduction}

In 2018 November Parker Solar Probe (PSP) became the first satellite mission to penetrate deep into the inner heliosphere, getting as close as 35.7 solar radii from the Sun. Between 2018 and 2024 this distance will progressively shrink to 9.8 solar radii $\left(R_{\odot}\right)$, offering unique opportunities to study in situ the young solar wind (Fox et al. 2016). The mission addresses two fundamental problems in space physics: coronal plasma heating and the acceleration of solar wind plasmas. In both problems wave-particle interactions involving magnetohydrodynamic (MHD) and kinetic-scale waves (including whistlers) are known to play an important role.

During its first solar encounter PSP was nearly co-rotating with the Sun for more than one week and was immersed in a slow but highly Alfvénic solar wind emerging from a small equatorial coronal hole (Bale et al. 2019; Kasper et al. 2019; Badman et al. 2020). As expected, in this type of solar wind the electron density and temperature increases with decreasing heliocentric distance, while the electron $\beta \mathrm{e}-$ the ratio of electron thermal pressure to magnetic pressure-drops (Halekas et al. 2020). The Strahl becomes narrower and dominates the suprathermal fraction of the distribution. Halekas et al. (2020) reported very low halo fractional densities near perihelion, much smaller than at larger heliocentric distances (McComas et al. 1992), smaller even than those previously reported at 0.3 au (Maksimovic et al. 2005; Štverák et al. 2009). The electron halo and Strahl evolve with increasing radial distance from the Sun, with the fraction of the distribution in the halo increasing, and the fraction of the distribution in the Strahl decreasing (Maksimovic et al. 2005; Štverák et al. 2009). These changes presumably are the result of wave-particle interactions on the electron distribution, which may transform the Strahl into the halo through scattering by wave-particle interaction processes. Wave perturbations are observed by PSP continuously in solar wind in the MHD frequency range (Chaston et al. 2020; Krasnoselskikh et al. 2020; Mozer et al. 2020a) and at higher frequencies (Malaspina et al. 2020; Mozer et al. 2020b). Malaspina et al. (2020) showed that higherfrequency plasma-wave power enhancements manifest themselves in predominantly electric field fluctuations near $0.7 f_{\mathrm{ce}}$ and near $1.0 f_{\text {ce }}$ with harmonics extending above $f_{\text {ce }}$. These waves were preliminarily identified as electrostatic whistlermode waves and electron Bernstein modes; their duration ranges from seconds to hours. Wave amplitudes significantly increase with decreasing distance to the Sun (Malaspina et al. 2020; Mozer et al. 2020b), suggesting that these waves play an important role in the evolution of electron populations in the near-Sun solar wind. Here we focus on electromagnetic waves in the $20-100 \mathrm{~Hz}$ frequency range that generally coincide with local perturbations of the magnetic field. As will be shown later, these are Doppler-shifted whistler waves.

One of the striking observations made by PSP during the first and third solar encounters is the omnipresence of rapid deflections of a magnetic field direction that is otherwise mostly radial. These so-called switchbacks are associated with 


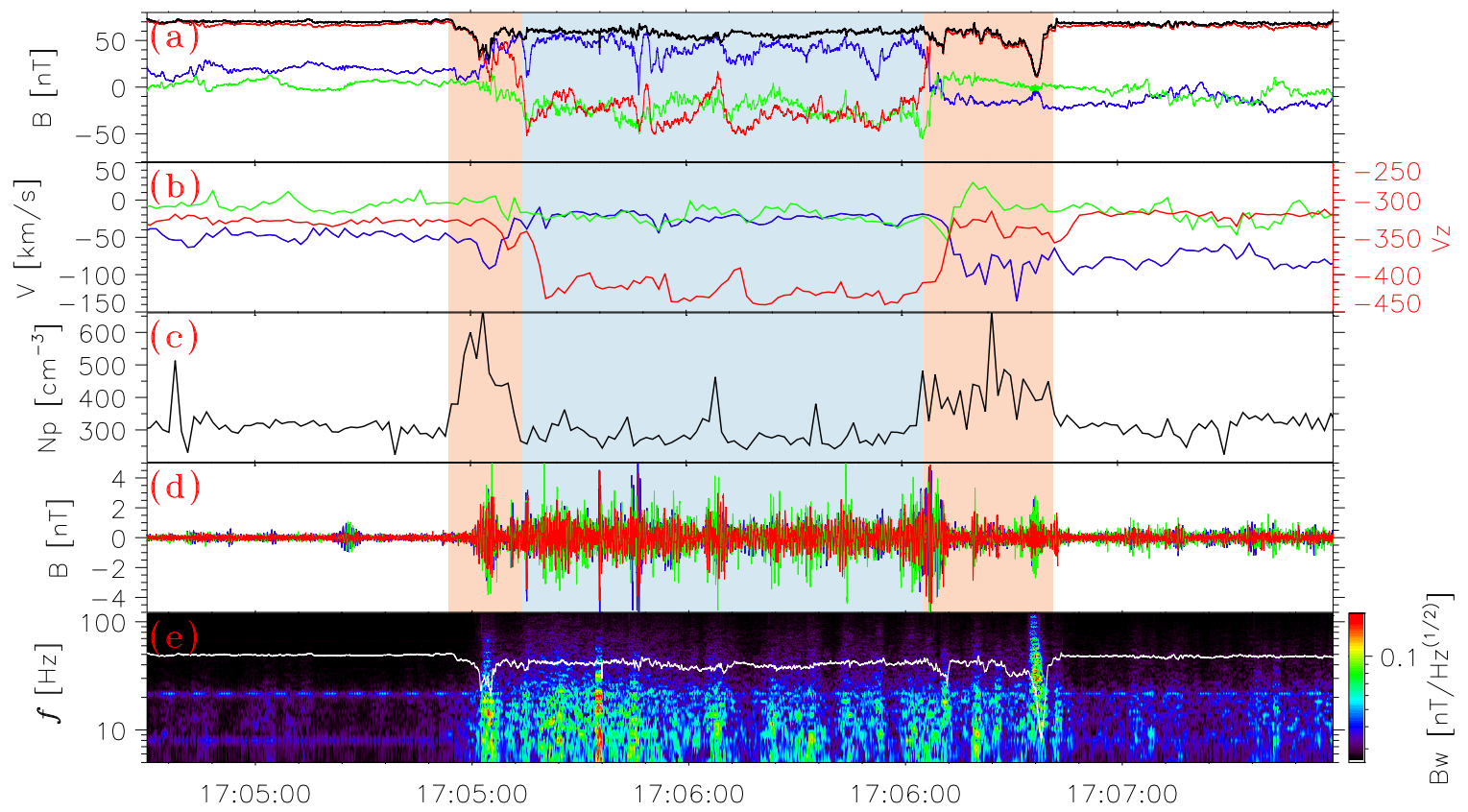

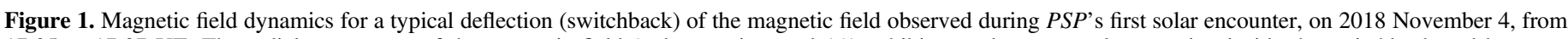

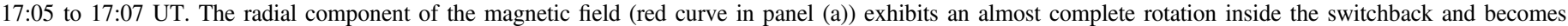

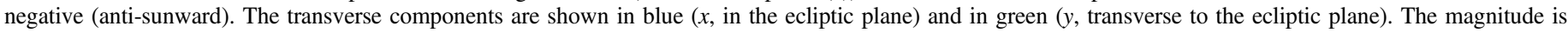

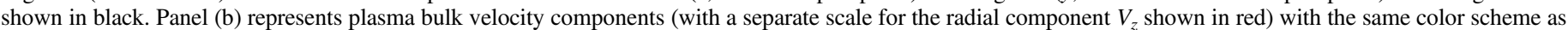

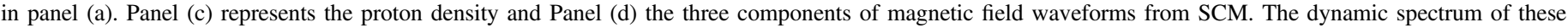
waveforms are shown in Panel (e), in which the solid white curve indicates the local lower hybrid frequency.

an enhanced radial bulk plasma velocity and strongly affect the dynamics of the magnetic field (Bale et al. 2019; Kasper et al. 2019; Dudok de Wit et al. 2020; Krasnoselskikh et al. 2020; Mozer et al. 2020a). Some lead to a complete reversal of the magnetic field, hence the name switchback. These deflections are observed during the first and second solar encounters, in slow but highly Alfvénic winds. They occur on timescales of seconds to hours and they are likely to be generated deep inside the corona (Dudok de Wit et al. 2020). Some switchbacks are accompanied by a small drop (of a few percent) in the amplitude of the magnetic field (Krasnoselskikh et al. 2020). The boundaries of these structures are plasma discontinuities that often have a significant normal component with respect to the magnetic field (Krasnoselskikh et al. 2020). Interestingly, they are accompanied by enhanced levels of wave activity (Krasnoselskikh et al. 2020; Mozer et al. 2020a).

Most of the waves that are observed near or during switchbacks belong to the MHD and whistler frequency ranges. However, low-frequency waves (with frequencies of a few $\mathrm{Hz}$ in the spacecraft frame) have also been observed; they have been identified as surface waves on the plasma discontinuities (Krasnoselskikh et al. 2020) and presumably are generated by surface velocity shift instabilities (Mozer et al. 2020a). In the following we concentrate on waves that belong to the whistler frequency range, motivated by the major impact whistler-mode fluctuations are known to have on energetic electrons. In the solar wind such waves affect the heat flux through the scattering of Strahl electrons (Kajdic et al. 2016), while in the Earth's magnetosphere they control the dynamics of the population of relativistic electrons (Horne 2007; Thorne 2010). Whistler waves in the solar wind have been studied in detail at 1 au (Lacombe et al. 2014) and, more recently, down to 0.3 au with HELIOS observations (V. K. Jagarlamudi 2020, private communication). Two potential sources of whistler waves in the solar wind are wave-particle interactions through electromagnetic instabilities and wavewave interactions (Saito \& Gary 2007). PSP provides us with a unique opportunity to study these waves much deeper in the inner heliosphere, in regions where, precisely, they may influence the electrons populations of the young solar wind.

\section{PSP Observations of Whistler Waves}

In the following, we investigate whistler waves by means of electric and magnetic field fluctuations. PSP measures magnetic fluctuations between DC and typically $30 \mathrm{~Hz}$ with the Fluxgate Magnetometer (MAG), and above typically $10 \mathrm{~Hz}$ with the Search-Coil Magnetometer (SCM). The electric field is measured by two pairs of electric field antennas (EFI). The outputs of SCM and EFI are sampled by the Digital Fields Board (DFB), which delivers a large variety of data products (Malaspina et al. 2016). All these instruments belong to the FIELDS consortium and are described in detail in Bale et al. (2016). In what follows, we concentrate on waveforms that are sampled at $292.97 \mathrm{~Hz}$ although spectral matrices are also available for probing higher frequencies. The proton density and velocity are derived from a Faraday cup that is a part of the SWEAP consortium (Kasper et al. 2016). These particle data are sampled every $12 \mathrm{~s}$.

The first perihelion pass of $P S P$ occurred on 2018 November 7 at a distance of 35.7 solar radii. During the 4-5 days that preceded and followed the perihelion the unperturbed magnetic field was directed mostly sunward with a magnitude of approximately 50-70 nT. The bulk velocity of the solar wind was in the range of $300-340 \mathrm{~km} \mathrm{~s}^{-1}$. A typical switchback structure that occurred on 2018 November 4 is illustrated in Figure 1. The reversal is best evidenced by the sudden change in sign of the radial component of the magnetic field, which is 

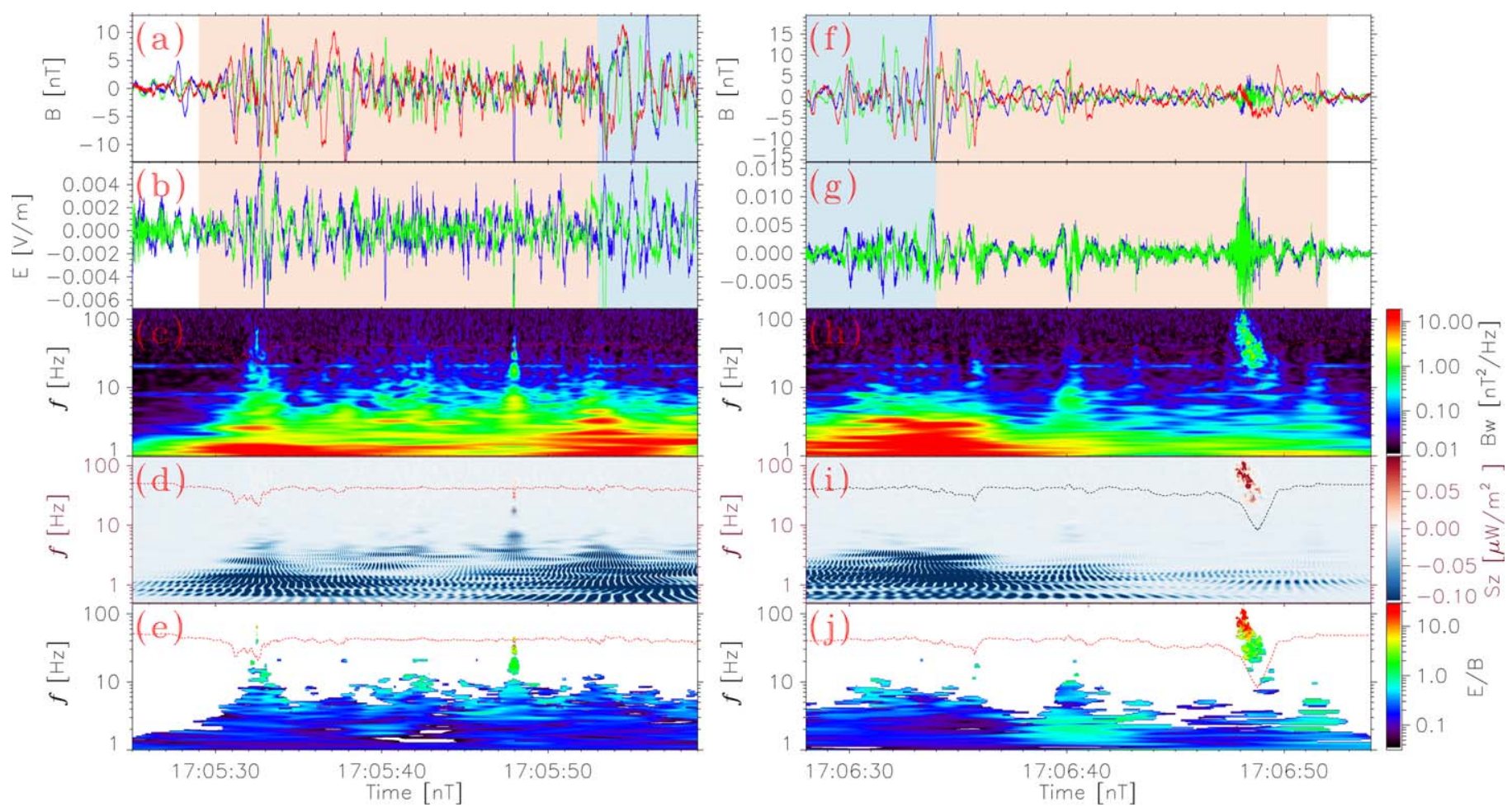

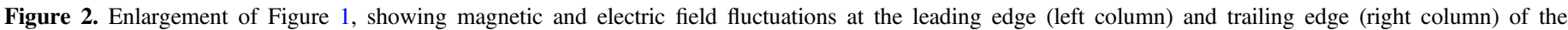

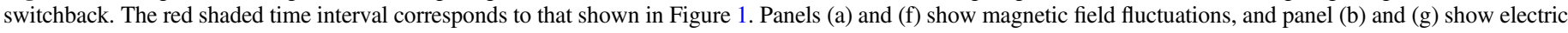

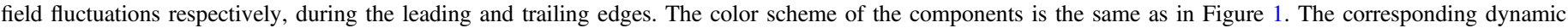

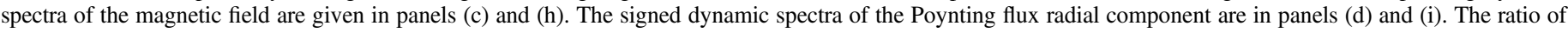
wave power of electric and magnetic field perturbations is in panels (e) and (j).

shown in red in Figure 1. For this particular event, which has been analyzed in detail by Krasnoselskikh et al. (2020), the magnetic field inside the switchback temporarily decreases from 70 to less than $50 \mathrm{nT}$. This structure has extended boundaries that last for several seconds; see Figure 1(a). Notice that the dip in the magnetic field amplitude does not coincide with the deflection; it starts approximately $10 \mathrm{~s}$ before the leading edge of the switchback and ends approximately $15 \mathrm{~s}$ after the trailing edge. These transition periods are marked with shaded bands in Figure 1.

Both the leading and trailing edges of the switchback are accompanied by a short but conspicuous dip in the amplitude of the magnetic field, which drops by $30 \mathrm{nT}$ (leading edge) and by 13 nT (trailing edge); these dips last for a few seconds. Both edges also coincide with an enhancement of the proton density, which rises from approximately 300 to $600 \mathrm{~cm}^{-3}$. Switchbacks are always accompanied by an increase in wave activity, which is well illustrated in Figure 1(d) by magnetic field fluctuations recorded by the SCM search-coil. Figure 1(e) shows the corresponding dynamic spectrum, which reveals broadband wave activity.

The local dip that occurs in the magnetic field at the extended leading edge of the switchback coincides with an enhancement of wave activity; see Figures 2(a) and (b). The frequency of these waves is in the MHD range, below the local proton gyrofrequency whose Doppler-shifted frequency is between 1 and $3 \mathrm{~Hz}$ (Figure 2(c)); the corresponding frequencies in the plasma rest frame are $0.3-0.5 \mathrm{~Hz}$. In that frequency range, the measured amplitude of the magnetic field reaches typically $10 \mathrm{nT}$, and the electric field $4 \mathrm{mV} \mathrm{m}^{-1}$. The radial component of the Poynting flux in the plasma frame is negative; i.e., it is directed anti-sunward as is usual for waves that are observed in the solar wind. The ratio of magnetic to electric field wave power (Figure 2(e)) agrees well with that of Alfvén waves with an effective antenna length of approximately $2.4 \mathrm{~m}$ (Mozer et al. 2020b).

The trailing edge of the switchback shown in Figure 2 reveals a large-amplitude surface wave-like perturbation whose magnetic amplitude reaches $0.3-0.4$ of the background field; see Figure 2(f). More details on the properties of these waves can be found in Krasnoselskikh et al. (2020). Notice in the local dip of the magnetic field a brief enhancement of higherfrequency wave activity that is best seen in the electric field where it reaches amplitudes as large as $15 \mathrm{mV} \mathrm{m}^{-1}$ (see Figure $2(\mathrm{~g})$ ), while in the magnetic field it goes up to $2 \mathrm{nT}$. In Figure 3 we enlarge this small dip to highlight its coincidence with the wave packet, whose frequency ranges from 20 to $120 \mathrm{~Hz}$; see Figures 2(h) and 3. Interestingly, this wave propagates sunward as the radial component of the Poynting flux is significantly positive (Figure 2(i)). Taking into account the Doppler shift, the frequencies in the plasma frame should be considerably higher and belong to the whistler frequency range. This is confirmed by the value of the electric and magnetic field wave power ratio $E_{w} / B_{w}$ in Figure 2(j), which is significantly greater than expected from the dispersion relation for such low-frequency waves.

In Figure 3 we zoom in the trailing edge of the same switchback and see that the local dip in the magnetic field is essentially caused by a decrease of its radial component. This dip coincides with an increase of the ratio between electron plasma frequency and electron gyrofrequency from 120 to approximately 500; see Figure 3(b). A polarization analysis 


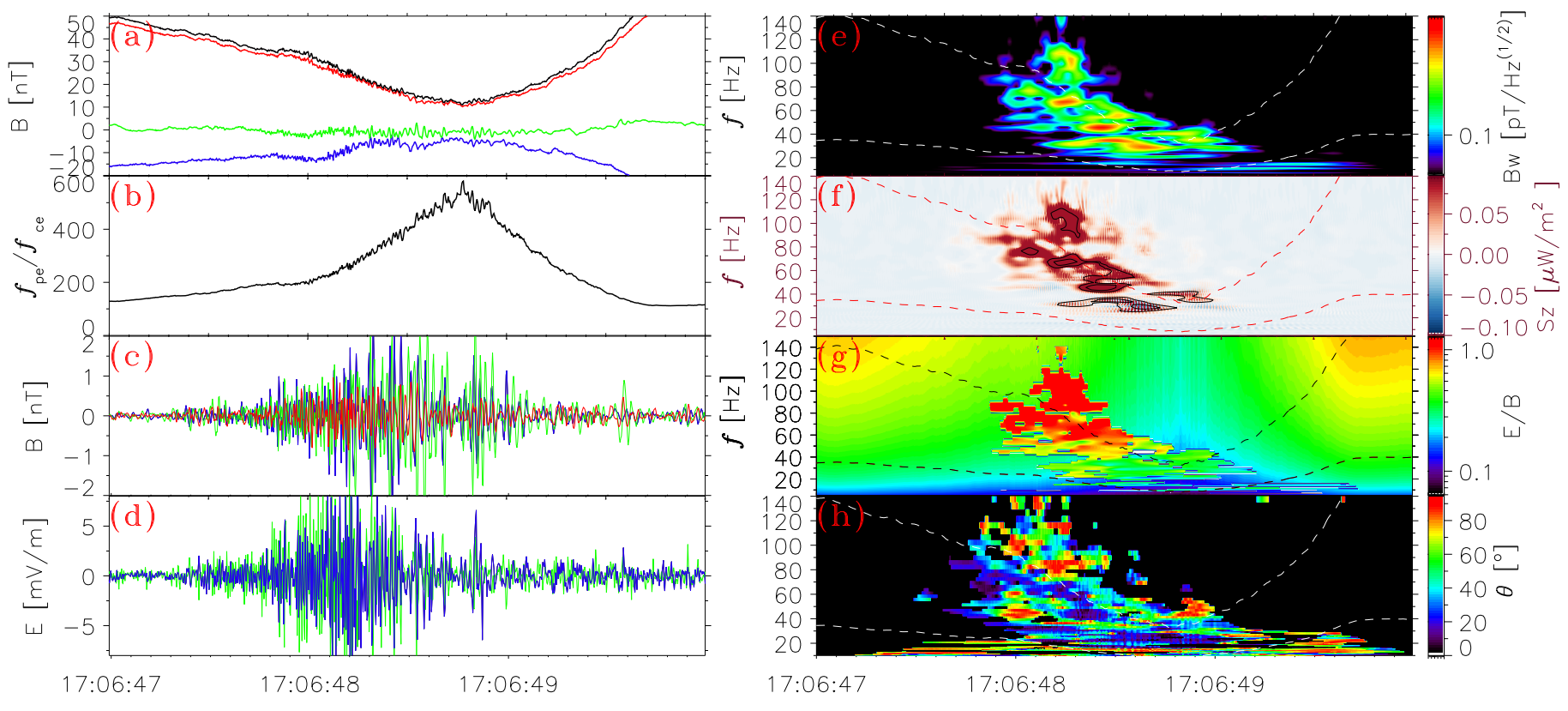

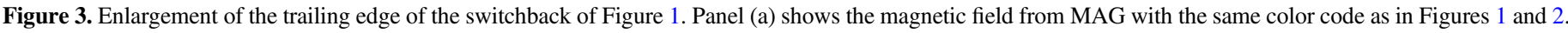

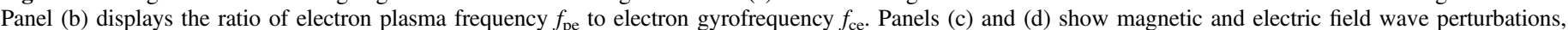

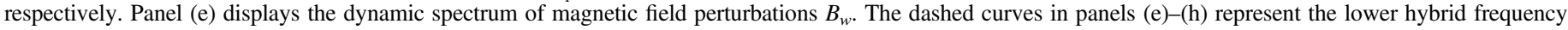

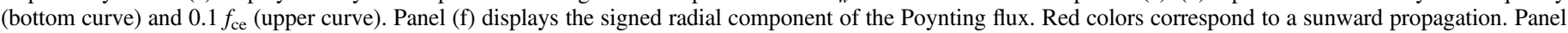

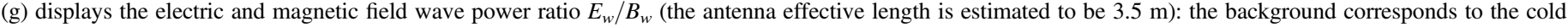

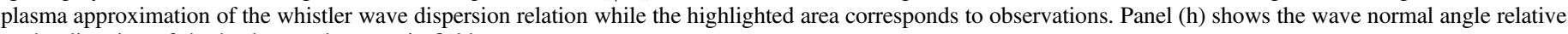
to the direction of the background magnetic field.

reveals a right-handed circular polarization of the magnetic field and an elliptical polarization of the electric field with a $\pi$ $/ 2$ phase shift. The dynamic spectrum in Figure 3(e) shows a complex inner structure of the wave packet, which consists of a series of bursts. The phase shift of the magnetic and electric field components transverse to the radial direction attest a sunward propagation. Notice how the sign of the radial component of the Poynting vector (Figure 3(f)) changes from positive (sunward) at high frequencies to negative (antisunward) at lower frequencies where, presumably, we have MHD waves. The frequencies of these wave packets fall between the lower hybrid frequency $f_{\mathrm{lh}}$ (lower dashed curve in Figures 3(f) and (g)) and one-tenth of the electron cyclotron frequency $f_{\text {ce }}$ (upper dashed curve), similarly to what is known for whistler waves near 1 au (e.g., Lacombe et al. 2014). From all these properties we conclude that these are whistler wave packets.

The dispersion relation for cold plasma whistler waves gives us an $E / B$ ratio that is significantly lower than the observed one, which appears highlighted in Figure $3(\mathrm{~g})$. This suggests that the observed frequency range of our whistler waves is shifted down by the Doppler effect as the whistler phase velocity (300-500 $\mathrm{km} \mathrm{s}^{-1}$ ) is comparable to that of the plasma bulk velocity. To evaluate this Doppler shift and reconstruct the real wave frequency we need to evaluate the wave normal angle relative to the background magnetic field direction (shown in Figure 3(h)) and the angle between the wave normal and the bulk velocity direction. The observed whistlers are found to have a wide range of wave normal angle values from quasiparallel propagation to quasi-electrostatic propagating close to the resonance cone corresponding to the complex structure of the dynamics spectrum (Figure 3(b)). Figure 3(h) thereby further supports the idea that our complex wave packet consists of a bunch of distinct and narrowband wave bursts.
We derive the wave frequency in the solar wind plasma frame from the Doppler shift and the whistler local parameters as obtained in the cold plasma approximation by making use of the wave normal angle values and the angle between wave normal and the bulk velocity direction. The reconstruction scheme is shown in Figure 4(a) and the spectrum in the plasma frame is presented in Figure 4(b). The resulting frequencies of the wave packet are found to be in the range of $100-350 \mathrm{~Hz}$, which corresponds to $0.2-0.5$ of the local electron gyrofrequency $f_{\text {ce }}$. Wave normal angles (Figure $3(\mathrm{~h})$ ) vary for different whistler subpackets from close to parallel propagation to oblique (close to the resonance cone) that presumably reflect the effect of the propagation in inhomogeneous background magnetic field. The values of the $E_{w} / B_{w}$ wave power ratio estimated for whistlers with the resulting higher frequency $\left(0.2-0.5 f_{\text {ce }}\right)$ are sufficiently higher $(\sim 3-5$ times $)$ than the estimated for the observed frequency of $0.05-0.1 f_{\text {ce }}$. While the observed whistler electric field (up to $10 \mathrm{mV} \mathrm{m}^{-1}$ ) is closer to whistler dispersion parameters for the restored (to the plasma frame) wave frequency, we find that it is still $\sim 3-4$ times above the values that are estimated from the dispersion relation from the observed magnetic field power. This might be explained by the higher effective length of the EFI (of typically up to $3.5 \mathrm{~m}$ ) at higher frequencies.

The entire discussion has so far been based on one single whistler packet; two other examples will be given below. These events, however, are representative of the numerous ones that were observed during PSPs first solar encounter. More than $90 \%$ of them coincide with local depressions of the magnetic field or with sudden deflections of the magnetic field. The latter do not have to be complete switchbacks because partial deflections of the magnetic field also frequently give rise to whistler wave packets as long as the deflection is sudden and has the same characteristics as a complete switchback. The 

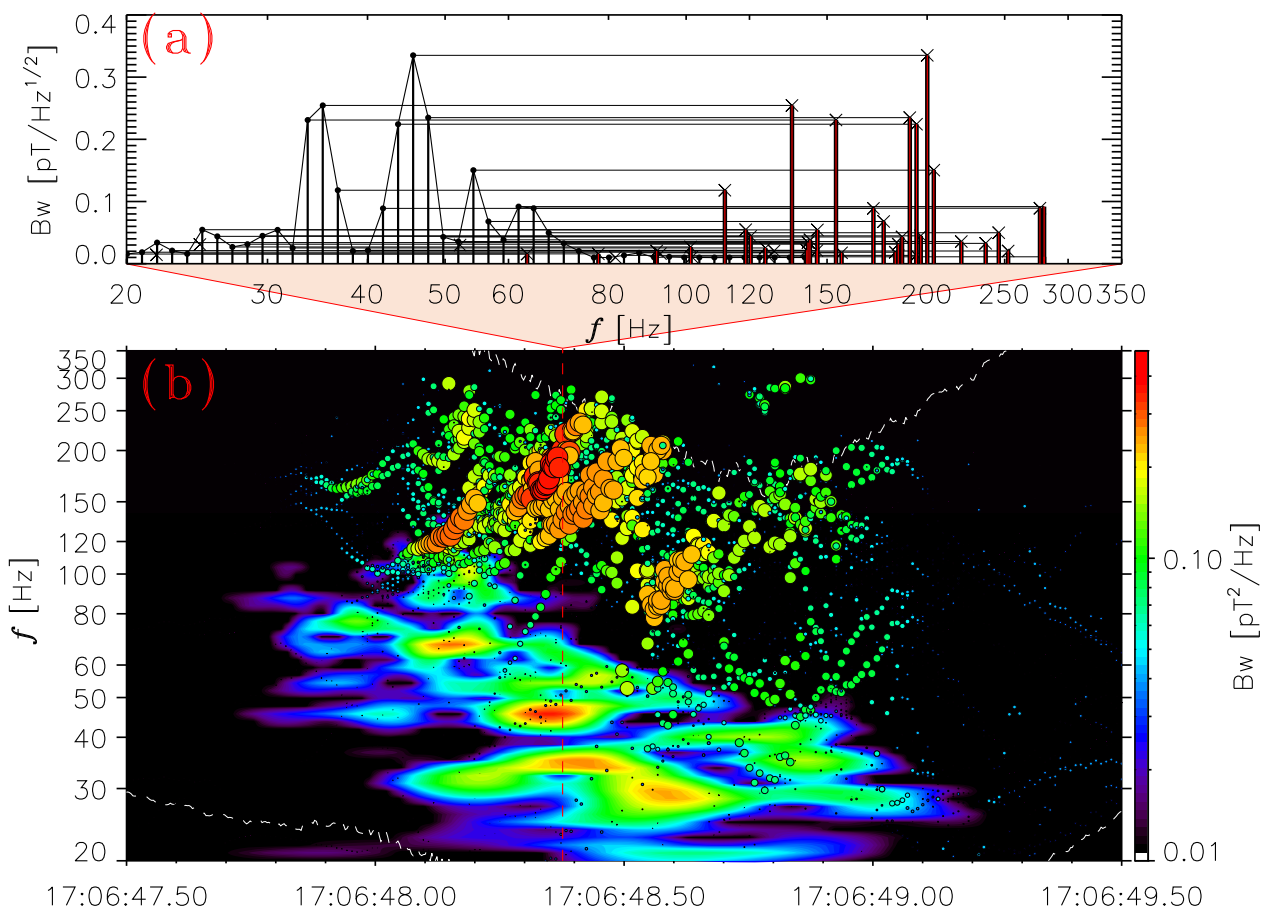

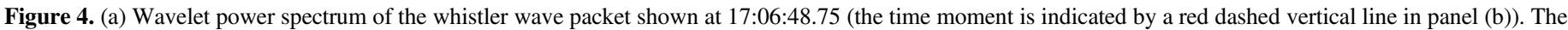

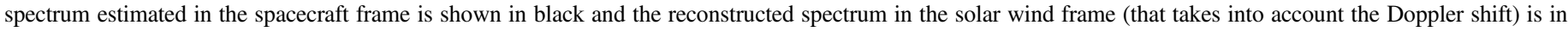

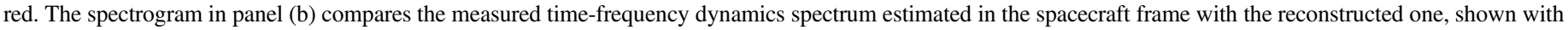
circles (wave amplitude is color-coded and indicated by the circles size).

number of whistlers per day varies considerably and reflects the large variability of the number of switchbacks. During the first encounter we typically observe between 20 and 50 events per day that are unambiguously identified as whistler waves. This rate of occurrence is considerably larger than what has recently been predicted from HELIOS observations of whistlers at different distances from the Sun greater than $0.3 \mathrm{au}(\mathrm{V}$. K. Jagarlamudi 2020, private communication).

\section{Discussion and Conclusion}

Let us now focus on the properties of the sunwardpropagating whistler wave bursts as observed by PSP during the first solar encounter. The analysis shows that waves observed in the $20-100 \mathrm{~Hz}$ frequency range are electromagnetic right-hand polarized whistlers propagating sunward both in the plasma frame and in the spacecraft frame; the value of their phase velocity value is usually higher than the bulk plasma velocity at 35.7 solar radii. These low-frequency electromagnetic whistler bursts are frequently associated with local minima of the background magnetic field magnitude. Two examples of whistler bursts (from the numerous cases captured on 2018 November 3-5 and having similar properties), both associated with local magnetic field magnitude minima, propagating sunward and captured on 2018 November 4 are presented in Figure 5.

The population of such sunward-propagating whistlers can efficiently scatter the energetic particles of the solar wind and affect the Strahl population to spread their field-aligned pitchangle distribution through pitch-angle scattering. The whistler resonance condition with electrons is given by $\omega-\boldsymbol{k} \boldsymbol{V}=\frac{n \Omega_{\mathrm{ce}}}{\gamma}$ where $\boldsymbol{k}$ is wave vector; $\Omega_{\text {ce }}=2 \pi f_{\text {ce }} ; \boldsymbol{V}$ is electron velocity, $n$ is an integer that can take on positive and negative values, and $\gamma$ is the Lorentz factor. For sunward-propagating whistlers with frequencies of $100-300 \mathrm{~Hz}$, the resonance conditions are realized (due to inhomogeneities of the background magnetic field magnitude) for electrons with velocities between 3000 and $20,000 \mathrm{~km} \mathrm{~s}^{-1}(\sim 50 \mathrm{eV}-1 \mathrm{keV})$, which covers the observed Strahl energy range (Halekas et al. 2020) and potentially leads to efficient wave-particle interactions producing local acceleration (Artemyev et al. 2013a; Kis et al. 2013) and scattering of the Strahl electrons. Such a scattering can be even more efficient when taking into account that a significant part of observed waves is oblique. Indeed, when the wave normal angles are found to be between the local Gendrin angle $\left(\cos \theta_{G}=2 f / f_{\text {ce }}\right.$, Gendrin 1961) and the local whistler resonance angle $\left(\cos \theta_{\text {res }}=f / f_{\text {ce }}\right)$, effective scattering is strongly enhanced (Artemyev et al. 2013b, 2014, 2016; Mourenas et al. 2014; Agapitov et al. 2014) on higher-order resonances. Such a scattering by high-amplitude whistler waves (whose amplitude reaches up to $10 \%$ of the background magnetic field magnitude) can regulate the heat flux as shown by RobergClark et al. (2019). For that reason the observed high-amplitude waves are likely to be an important factor in the dynamics of the solar wind distribution (Roberg-Clark et al. 2018a, 2018b). The fraction of energetic electrons that belong to the halo distribution increases with the distance from the Sun while the fraction of Strahl population decreases (Maksimovic et al. 2005; Štverák et al. 2009; Halekas et al. 2020), which suggests a gradual transformation of the Strahl into the halo, presumably by pitch-angle scattering. Meanwhile, the angular width of the Strahl increases with radial distance (Hammond et al. 1996; Graham et al. 2017; Berčič et al. 2019). The whistler amplitudes that have been observed by PSP near the Sun are sufficiently larger than those observed 1 au (Breneman et al. 2010; Lacombe et al. 2014; Stansby et al. 2016; Tong et al. 2019a, 2019b). Their generation mechanism is presumably related to the cyclotron instability guided by a transverse 

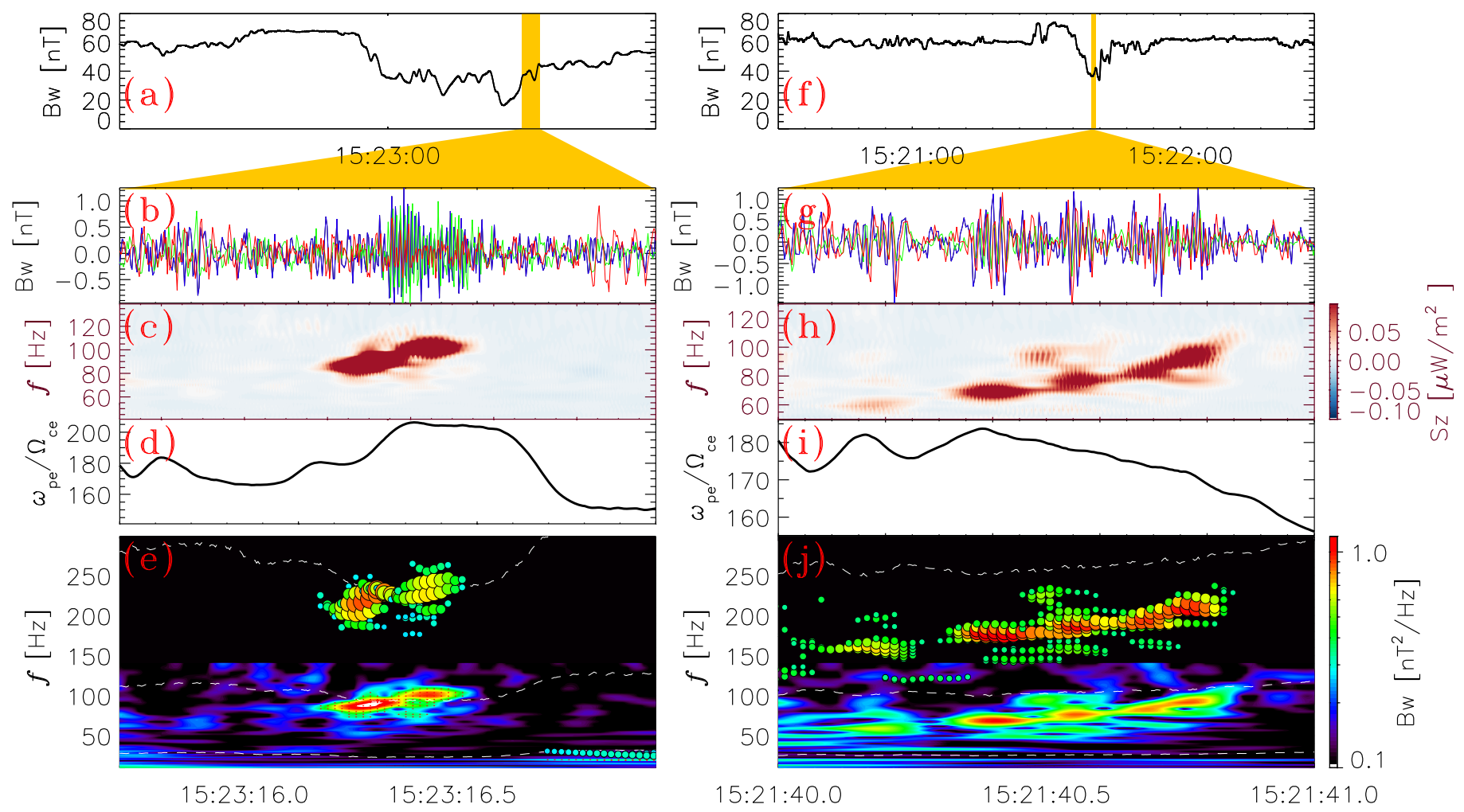

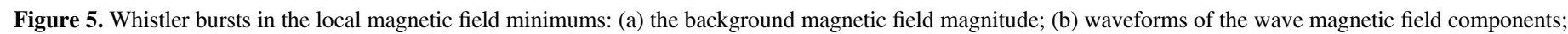

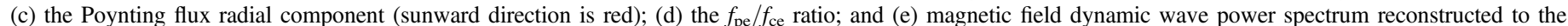
plasma frame power spectrum is shown by the circles with color-coded by wave amplitude. Panels (f)-(j) represent a similar case.

temperature anisotropy of $\sim 200 \mathrm{eV}$ electrons and can be triggered by a magnitude gradient around the magnetic field magnitude minimum; this will be the subject for a future study (involving the electron distribution function processing). The statistical studies by Tong et al. (2019b) showed a coincidence between the presence of whistlers and periods of higher temperature anisotropy. Numerical studies indicate electron beams as a possible source for whistler wave generation (Agapitov et al. 2015; Mourenas et al. 2015; Li et al. 2016; Kuzichev et al. 2019; Roberg-Clark et al. 2019).

To conclude:

(1) PSP observations of electromagnetic whistler wave packets in the solar wind at $\sim 35.7 R_{\odot}$ have revealed the existence of low-frequency (with frequencies of $20-80 \mathrm{~Hz}$ in the spacecraft frame, which is below 0.1 $f_{\text {ce }}$ ) whistler wave packets. These waves coincide with local minima of the magnetic field magnitude or with edges of magnetic switchbacks.

(2) These whistler waves are found to propagate sunward. Their phase velocity is in the range of $300-500 \mathrm{~km} \mathrm{~s}^{-1}$, which leads to a significant Doppler frequency downshift from 200 to $300 \mathrm{~Hz}$ in the solar wind frame to $20-80 \mathrm{~Hz}$ in the spacecraft frame. This downshift allows these waves to be resolved by waveforms from magnetic (SCM and MAG) and electric (EFI) sensors, which are sampled at $292.97 \mathrm{~Hz}$ at perihelion.

(3) The whistler frequency in the plasma frame is of the order of $0.2-0.5$ of the local electron gyrofrequency $f_{\text {ce }}$.

(4) The polarization of these waves varies in different wave packets from quasi-parallel to significantly oblique and close to the resonance values of wave normal angle (presumably due to the propagation in inhomogeneous background magnetic field).

(5) The wave amplitude reaches $2-4 \mathrm{nT}$, which corresponds to up to $10 \%$ of the background magnetic field. This amplitude is much larger than what is observed in the solar wind at $1 \mathrm{au}$ (Lacombe et al. 2014; Stansby et al. 2016; Tong et al. 2019a, 2019b; Vasko et al. 2019). Such waves are very effective in scattering the Strahl population of solar wind electrons as shown recently in numerical simulations by Roberg-Clark et al. (2019). We conjecture that these whistler waves play a significant role in scattering the Strahl population and breaking the heat flux in the inner heliospheric solar wind.

O.V.A. and J.F.D. were supported by NASA grant 80NNSC19K0848; O.A. was partially supported by NSF grant no. 1914670 and NASA Living with a Star (LWS) program (contract 80NSSC20K0218); T.D., V.K., C.F., and A.L. acknowledge the support of CNES and SDB the support of the Leverhulme Trust Visiting Professorship program. Parker Solar Probe was designed, built, and is now operated by the Johns Hopkins Applied Physics Laboratory as part of NASA's Living with a Star (LWS) program (contract NNN06AA01C). Support from the LWS management and technical team has played a critical role in the success of the Parker Solar Probe mission. All the data used in this work are available on the FIELDS data archive (http://fields.ssl.berkeley.edu/data/).

\section{ORCID iDs}

O. V. Agapitov (i) https://orcid.org/0000-0001-6427-1596

T. Dudok de Wit (iD https://orcid.org/0000-0002-4401-0943

F. S. Mozer (iD https://orcid.org/0000-0002-2011-8140 
J. F. Drake (1) https://orcid.org/0000-0002-9150-1841

D. Malaspina (1) https://orcid.org/0000-0003-1191-1558

V. Krasnoselskikh (i) https://orcid.org/0000-0002-6809-6219

S. Bale (1) https://orcid.org/0000-0002-1989-3596

P. L. Whittlesey (1) https://orcid.org/0000-0002-7287-5098

A. W. Case (1) https://orcid.org/0000-0002-3520-4041

C. Froment (i) https://orcid.org/0000-0001-5315-2890

J. C. Kasper (1) https://orcid.org/0000-0002-7077-930X

K. E. Korreck (1) https://orcid.org/0000-0001-6095-2490

R. J. MacDowall (1) https://orcid.org/0000-0003-3112-4201

M. Pulupa (i) https://orcid.org/0000-0002-1573-7457

M. Stevens (i) https://orcid.org/0000-0002-7728-0085

\section{References}

Agapitov, O. V., Artemyev, A. V., Mourenas, D., et al. 2014, JGRA, 119, 1606 Agapitov, O. V., Artemyev, A. V., Mourenas, D., Mozer, F. S., \& Krasnoselskikh, V. 2015, GeoRL, 42, 10140

Artemyev, A., Agapitov, O., Mourenas, D., et al. 2016, SSRv, 200, 261

Artemyev, A. V., Agapitov, O. V., \& Krasnoselskikh, V. V. 2013a, PhPl, 20, 124502

Artemyev, A. V., Mourenas, D., Agapitov, O., et al. 2013b, AnGeo, 31, 599 Artemyev, A. V., Vasiliev, A. A., Mourenas, D., et al. 2014, GeoRL, 41, 5727 Badman, S. T., Bale, S. D., Martínez Oliveros, J. C., et al. 2020, ApJS, 246, 23 Bale, S. D., Badman, S. T., Bonnell, J. W., et al. 2019, Natur, 576, 237

Bale, S. D., Goetz, K., Harvey, P. R., et al. 2016, SSRv, 204, 49

Berčič, L., Maksimović, M., Landi, S., \& Matteini, L. 2019, MNRAS, 486, 3404

Bowen, T. A., Mallet, A., Huang, J., et al. 2020, ApJS, 246, 66

Breneman, A., Cattell, C., Schreiner, S., et al. 2010, JGRA, 115, A08104

Chaston, C., Bonnell, J. W., Bale, S. D., et al. 2020, ApJS, in press (doi:10. 3847/1538-4365/ab745c)

Dudok de Wit, T., Krasnoselskikh, V., Bale, S. D., et al. 2020, ApJS, 246, 39

Fox, N. J., Velli, M. C., Bale, S. D., et al. 2016, SSRv, 204, 7

Gendrin, R. 1961, P\&SS, 5, 274

Graham, G. A., Rae, I. J., Owen, C. J., et al. 2017, JGRA, 122, 3858

Halekas, J. S., Whittlesey, P., Larson, D. E., et al. 2020, ApJS, 246, 22
Hammond, C. M., Feldman, W. C., McComas, D. J., Phillips, J. L., \& Forsyth, R. J. 1996, A\&A, 316, 350

Horne, R. B. 2007, NatPh, 3, 590

Kajdic, P., Alexandrova, O., Maksimovic, M., Lacombe, C., \& Fazakerley, A. N. 2016, ApJ, 833, 172

Kasper, J. C., Abiad, R., Austin, G., et al. 2016, SSRv, 204, 131

Kasper, J. C., Bale, S. D., Belcher, J. W., et al. 2019, Natur, 576, 228

Kis, A., Agapitov, O., Krasnoselskikh, V., et al. 2013, ApJ, 771, 4

Krasnoselskikh, V., Larosa, A., Agapitov, et al. 2020, ApJS, submitted

Kuzichev, I. V., Vasko, I. Y., Soto-Chavez, A. R., et al. 2019, ApJ, 882, 81

Lacombe, C., Alexandrova, O., Matteini, L., et al. 2014, ApJ, 796, 5

Li, W., Mourenas, D., \& Artemyev, A. V. 2016, GeoRL, 43, 8867

Maksimovic, M., Zouganelis, I., Chaufray, J.-Y., et al. 2005, JGRA, 110, A09104

Malaspina, D., Ergun, R. E., Bolton, M., et al. 2016, JGRA, 121, 5088

Malaspina, D., Halekas, Laura Berčič, et al. 2020, ApJS, 246, 21

McComas, D. J., Bame, S. J., Feldman, W. C., Gosling, J. T., \& Phillips, J. L. 1992, GeoRL, 19, 1291

Mourenas, D., Artemyev, A., Agapitov, O., et al. 2014, JGRA, 119, 2775

Mourenas, D., Artemyev, A. V., Agapitov, O. V., Krasnoselskikh, V., \& Mozer, F. S. 2015, JGRA, 120, 3665

Mozer, F. S., Agapitov, O. V., Bale, S. D., et al. 2020a, ApJS, 246, 68

Mozer, F. S., Agapitov, O. V., Bale, S. D., et al. 2020b, ApJS, 246, 50

Roberg-Clark, G. T., Agapitov, O., Drake, J. F., \& Swisdak, M. 2019, ApJ, 887,190

Roberg-Clark, G. T., Drake, J. F., \& Reynolds 2018a, PhRvL, 120, 035101

Roberg-Clark, G. T., Drake, J. F., Swisdak, M., \& Reynolds, C. S. 2018b, ApJ, 867,154

Saito, S., \& Gary, S. P. 2007, JGR, 112, A06116

Stansby, D., Horbury, T. S., Chen, C. H. K., \& Matteini, L. 2016, ApJL, 829, L16

Štverák, Š., Maksimovic, M., Trávníček, P. M., et al. 2009, JGRA, 114, A05104

Thorne, R. M. 2010, GeoRL, 372, 22107

Tong, Y., Vasko, I. Y., Pulupa, M., et al. 2019a, ApJL, 870, L6

Tong, Y., Vasko, I. Y., Artemyev, A. V., Bale, S. D., \& Mozer, F. S. 2019b, ApJ, 878, 41

Vasko, I. Y., Krasnoselskikh, V., Tong, Y., et al. 2019, ApJL, 871, L29 